\title{
Mga karanasan ng mga guro sa baitang 3 sa pagtuturo ng mother tongue gamit ang Ortograpiyang Waray ng Komisyon sa Wikang Filipino
}

\author{
De Mesa, Alvin $\bowtie$ \\ Leyte Normal University, Philippines (ar_demesa@ymail.com)
}

Received: 5 November 2021

Available Online: 2 January 2022
Revised: 29 November 2021 DOI: $10.5861 /$ ijrse.2022.208

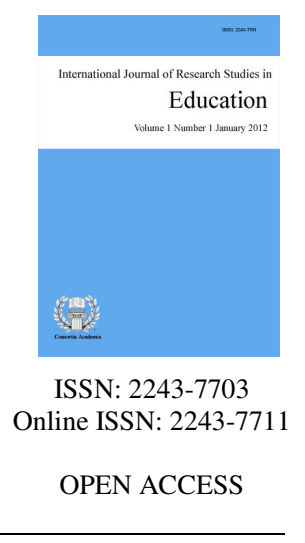

\section{Abstract}

This study aimed to determine the lived experiences of the Grade 3 teachers in teaching Mother Tongue using the Waray Orthography of the Filipino Language Commission. Utilizing the descriptive qualitative design and purposive sampling technique, the qualified participants of the study were identified. Participants were the teachers who received the copy of the Waray Orthography from the office of the Leyte Normal University-Center for Language and Culture, during the Regional Seminar entitled "Mga Saliksik sa Wikang Waray at iba pang Katutubong Wika" in celebration of the 2019 Filipino Language Month. On March 2021, the Focused Group Discussion (FGD) was conducted by the researcher through messenger, e-mails, and google meet. Availability of the participants was also considered and a consent letter was given to them for approval to participate in the study. Based on the results, it showed that before having the copy of the Waray Orthography, Grade 3 teachers experienced difficulty in teaching Mother Tongue, because they did not understand most of the words written in their reference module even if they are native speakers of Waray; but sometimes they heard it from their ancestors. However, after the participants received the copies of the Waray Orthography, they found it easy to teach Mother Tongue because the orthography helped them in identifying the Waray grapheme, syllable, spelling in writing/orally, consonant blends, digraph, and punctuation marks. Lastly, the participants were able to make their own Mother Tongue materials for Grade 3 based on the Waray Orthography of the Filipino Language Commission.

Keywords: lived experiences, mother tongue, Waray orthography, Grade 3 teachers, Filipino language commission 


\section{Mga karanasan ng mga guro sa baitang 3 sa pagtuturo ng mother tongue gamit ang Ortograpiyang Waray ng Komisyon sa Wikang Filipino}

\section{Introduksyon}

Sinasabing wika ang salamin ng kultura sa isang lugar na gumagamit nito. Wika ang paraan upang magkabuklod-buklod ang bawat tao. Iba-iba man ang wika, kailangan pa rin ang kaisahan ng wikang ginagamit ng bawat lugar. Maituturing din na ang mother tongue ang pinakapangunahing batayan sa pagkakaroon ng wikang panlahat sa komunidad, sa isang, lugar, o sa isang bansa.

Nagkakaroon lamang ng wikang panlahat kung mayroong matibay na batayan na paghuhugutan ng ano mang mother tongue. Upang magkaroon ng kaisahan, kailangan mayroon itong Ortograpiya na umaayon sa wikang panlahat o Wikang Pambansa. Dahil dito, maraming iba-ibang saloobin sa pagtuturo ng mother tongue na dahilan para magkaroon ng iba-ibang karanasan ang mga guro sa Baitang 3 sa pagtuturo ng mother tongue. Lumabas sa mga panawagan ng mga guro na mabisang karanasan sa kanila kung ang pamahalaan ay maglalaan ng pondo upang magsagawa ng mga pagsasanay kaugnay sa pagtuturo ng mother tongue. Sang-ayon dito ang UNESCO (2019) na mainam ang iba't ibang worksyap na gagawin para mas mapaunlad pa ang pagtuturo ng mother tongue, dahil naniniwala sila na magsisilbi itong gabay sa mga guro upang hindi sila mahirapan sa pagtuturo ng mother tongue. Samantalang si Jackson (2015) ay nagpasaring na magiging madali lamang ang pagtuturo ng mother tongue kung napaghandaan ito ng pamahalaan, ahensiya ng gobyerno, at ng pamahalaan mismo upang sa gayon maging maganda ang karanasan sa pagtuturo ng mga guro.

Sa isinagawang pagbeberipika nina Home (2015), ang ortograpiyang Filipino ay nagiging mabisa kung ang pagbigkas at bokabularyo nito ay nauunawaan ng bawat gumagamit ng wikang ito. Samantala, binanggit ni Cabigao (2012), na hindi magkakaroon ng problema ang isang ortograpiya partikular na sa Ortograpiyang Filipino kung ito ay ginagamit sa controlling domains gaya ng pamahalaan, sa mababa at mataas na antas ng pagkatuto, iba't ibang propesyon, komersiyo, at iba pa. Kung gayon, kailangan manguna ang pamahaalang lokal na tangkilikin ang mother tongue. Dapat din silang magsagawa ng mga hakbang na may mangangalaga ng kanilang sariling wika lalo pa at ang Baitang 3 ay nasa Basic Education kung saan dapat suportado ito ng pamahalang lokal. Dagdag pa, bahagi ng mandato ng Komisyon sa Wikang Filipino (KWF) ang pagbuo at pagsasaayos ng ortrograpiya (kung mayroon man) ng mga katutubong wika (mother tongue) sa Pilipinas na ginagamit ng mga tao (Corrige at KWF, 2018).

Dahil sa mainit na usapin kung papaano ituturo ang mother tongue lalo pa at walang opisyal na Ortograpiyang Waray, gumawa ng paraan ang Komisyon sa Wikang Filipino sa pangunguna ni Gng. Corrige upang maisakatuparan ito at matamasa na ng mga Waray ang sariling ortograpiya na nakaankla sa Ortograpiyang Filipino. Dumaan ito sa mahabang proseso at nagsagawa sila ng konsultasyon sa Samar, Leyte, at Biliran na dinaluhan ng mga guro, punongguro, ilang mamamayan ng rehiyon, at administrador ng paaralan kung saan ang huling konsultasyon ay isinagawa sa Leyte Normal University, Accreditation Evaluation Room, Lungsod Tacloban noong 08 Nobyembre 2017 (De Mesa, 2017). Sa pagsisikap ng KWF na mailathala at maipamahagi sa mga Waray ang mga nabuo nilang Ortograpiyang Waray, agad itong ipinamahagi at isinabay sa isinagawang Panrehiyong Seminar at Paglulunsad ng Ortograpiyang Waray na may temang Malayuning Pananaliksik sa Espisipikong Larang na ginanap sa Leyte Normal University noong 18-19 Agosto 2018 sa pangunguna ni Kgg. Virgilio S. Almario kasama sina G Evie Duclay at Komisyoner ng Rehiyon VIII, Kgg. Gerry B. Gracio (De Mesa, 2018).

Tunay na masasandalan ang isang batayang kagamitan sa pagtuturo partikular na sa mother tongue upang mapagkaisa ang posibleng karanasan ng mga guro na nagtuturo nito. Gaya nga ng ibinahagi ni Del Ayre (https://kwf.gov.ph/wp-content/uploads/ Ortograpiyang_Pambansa_1.pdf), ang ortograpiya ay mula sa dalawang 
Mga karanasan ng guro sa baitang 3 sa pagtuturo ng mother tongue gamit ang Ortograpiyang Waray

salita na sa una ay ortho na ibig sabihin sa wikang Griyego ay wasto, samantala, ang ikalawa ay graphia na ibig sabihin sa wikang Griyego o Latin ay pagsulat. Sa pagkakatong ito, ang Ortograpiyang Waray ng KWF ay magsisilbing batayan sa tamang pagsulat ng mga salita sa wikang Waray o sa mother tongue na Waray.

Upang makunan ng mga impormasyon kaugnay sa naging silbi ng Ortograpiyang Waray na naipamahagi sa mga guro na nagtuturo sa Baitang 3, minabuting inalam ng mananaliksik ang mga sumusunod na layunin sa isinagawang pag-aaral: 1. Ano ang karanasan ng mga guro sa Baitang 3 na nagtuturo ng Mother Tongue bago pa man nabuo ang Ortograpiyang Waray ng KWF? 2. Ano ang karanasan ng guro guro sa Baitang 3 na nagtuturo ng Mother Tongue pagkatapos matanggap ang kopya ng nabuong Ortograpiyang Waray ng KWF? At. 3. Ano ang implikasyon ng pagkakaroon ng Ortograpiyang Waray na binuo ng KWF sa pagtuturo ng Mother Tongue?

\section{Metodolohiya}

Ginamit ang disenyong Penominolohikal na Kwalitatibong pag-aaral upang matukoy ang mga karanasan ng mga guro sa pagtuturo ng Mother Tongue sa Baitang 3. Dahil sa penominal na karanasan ang pagtuturo ng Mother Tongue sa panahon na hindi pa ganap na nagkaroon ng batayang Ortograpiya sa wikang Waray, minabuting pinagtuunan ito ng pansin sa isinagawang pananaliksik. Dagdag pa rito, pinaniniwalaan ng maraming dalubhasa na si Edmund Husserl noong 1859-1938 na nakatuklas ng penominolohikal na paraan sa pananalliksik ay hindi rin nakaiwas sa mga puna na mahalaga ring isaalang-alang ang mga subhektibong pananaw ng mga tao katulad ng kanilang mga karanasan sa pang-araw-araw nilang pamumuhay (Zahavi, 2003). Kaya naman, mahigpit na pinagtuunan ng pansin sa isinagawang pag-aaral ang mga karanasan ng mga guro sa pagtuturo ng Mother Tongue na Waray at naging malaya ang mga kalahok na guro na sagutin ang ano mang katanungan na ibinigay sa kanila.

Samantala, purposive sampling naman ang ginamit sa pag-aaral na ito upang malayang mapili ng mananaliksik ang kailangan at kwalipikadong mga kalahok. Kaya naman, ang mga napiling mga naging kalahok ay mga direktang nabigyan lamang ng kopya na itinuturing na kwalipikadong kalahok sa isinagawang pag-aaral kung saan isinaalang-alang din ang etikal na konsiderasyon. Sa apatnapung nabigyan ng kopya, tanging labinlima (15) lamang ang kinilala at kwalipikadong mga kalahok dahil sila lamang ang nakapagbigay na angkop na tugon sa mga impormasyong kailangan ng mananaliksik habang ang iba naman ay nagtuturo ng ibang baitang. Kasama na rin sa pagtukoy ng mga kalahok ang mga gumagamit lamang ng Wikang Waray. Gamit ang platform na messenger at google meet ay nakapanayam ng mananaliksik ayon sa kanilang abeylabol na oras at napagkasunduang iskedyul ng pakikipanayam. Tanging ang mananaliksik lamang ang nagsilbing instrumentong ginamit sa isinagawang pag-aaral na may gabay sa pakikipanayam upang hindi makalimutan at magkaroon $\mathrm{ng}$ direksiyon ang isinagawang pakikipanayam o interbiyu.

\section{Resulta at Diskusyon}

Napag-alaman sa isinagawang pananaliksik na ang bawat suliranin ay nakitaan ng bawat tema. Tunghayan ang mga sumusunod: Una, Karanasan ng mga guro sa Baitang 3 na nagtuturo ng Mother Tongue BAGO pa man nabuo ang Ortograpiyang Waray ng KWF.

Nahirapan. Ito ang nabuong tema sa unang suliranin dahil naging karaniwang tugon ito ng mga kalahok. Nahirapan daw sila sa pagtuturo dahil sa hindi nila alam kung ano ang kanilang pagbabatayan sa pagtukoy ng tamang pagbigkas at pagsulat ng mga salita sa Mother Tongue na Waray.

Makikita sa Table 1, ang mga naging tugon ng mga gurong kalahok kaugnay sa kanilang karanasan sa pagtuturo ng Mother Tongue BAGO pa man nabuo ang Ortograpiyang Waray ng KWF. Makikita sa loob ng kahon ang mga tugon ng mga gurong kalahok na nagpatotoo sa nabuong tema kaugnay sa karanasan ng mga guro sa Baitang 3 na nagtuturo ng Mother Tongue bago pa man nabuo o nakatanggap ng kopya ng Ortograpiyang Waray mula sa KWF. 


\section{Table 1}

Pahayag na tugon ng mga kalahok sa temang nahirapn bago matanggap ang Ortograpiyang Waray ng KWF

\begin{tabular}{ll}
\hline \multicolumn{1}{c}{ Tugon ng mga gurong Kalahok } & \multicolumn{1}{c}{ Tema } \\
\hline Napakahirap sa parte namin, kasi wala kaming ibang pinagkukunan. & Napakahirap \\
Mahirap. Mayroon mang ibang materyales, hindi ko naman maintindihan. & Mahirap \\
A, napakahirap talaga! Mantakin mong isasalang ka dito na walang pagsasanay na ibinigay. & Napakahirap \\
Oo, sinasabi nila nariyan na ang modyul, pero naman, sobrang hirap unawain & Sobrang hirap \\
Ay, oo. Totoo nga masarap magturo ng sariling wika, pero naman mahirap ang mga Salita. & Mahirap \\
Sa akin, noong una akala ko, madali lang kasi Mother Tongue ko ito, mahirap pala. & Mahirap \\
Nutuwa ako, kasi wika ko, ituturo ko, iyon pala noong tumatagal na, nahihirapan na ako. & Nahihirapan \\
Madali sana kung nagbigay lamang ng pagsasanay, kaya masasabi kong nahirapan ako & Nahirapan \\
Natakot ako. Mahirap kasi ang mga salitang nakapaloob sa modyul. & Mahirap \\
Naawa ako sa mga bata, kasi may pagkakataon na mahirap talaga ang mga salita. & Mahirap \\
Mahirap ang mga salita, minsan sinabi sa akin ng aking mag-aaral, bakit daw hindi ko alam. & Mahirap \\
Diyos ko! Ayoko nang magsalita, mahirap talagang ituro & Mahirap \\
Siyempre kailangan magturo sa kabila ng kahirapan ng mga salitang ginamit. & Mahirap \\
Natakot dahil walang diksyunaryo, kaya nahirapan ako. & Nahirapan \\
Nag-alangan ako, hindi kasi pamilyar ang mga salita, kaya mahirap. & Mahirap \\
\hline
\end{tabular}

Napatotohanan ang pahayag ng UNESCO (2019) na mahalagang maglaan ng panahon ang ahensiya ng pamahalaan para sa isang worksyap kaugnay sa pagtuturo ng Mother Tongue upang hindi mahirapan ang mga guro sa pagtuturo nito. Bagamat maraming nabasa ang mananaliksik ng iba't ibang memoramdum ng DepEd tungkol sa pagsasagawa ng pagsasanay sa pagtuturo ng Mother Tongue, masasabing napakarami pa ring mga guro ang hindi nabigyan ng pagkakataon na makasali rito.

Subalit, kung susuriin ang mga naging tugon ng mga gurong kalahok, kailangan talaga nila ng mga materyales na magiging batayan kung sakaling mahirapan sila sa pagtuturo ng Mother Tongue na Waray. Para sa kaalaman ng lahat na mambabasa ng pananaliksik na ito, marami ring guro ang hindi taal na Waray na dahilan upang hindi nila lubos na maunawaan ang mga salita nito. Kaya naman, ang ginagawa nila ay puspusang pakiusap sa kapwa nila guro na nakauunawa ng wikang Waray. Sa kasamaang palad din, marami rin sa mga ito ang hindi rin alam kung ano ang ibig sabihin ng mga salitang ginamit sa modyul dahil tanging iilan na lamang na mga matatanda ang nakauunawa sa mga salitang ito na sinang-ayunan ni Cabigao (2015).

Samantala, matutunghayan ang ikalawang suliranin ng isinagawang pag-aaral kung saan nagbigay rin ng karanasan ang mga gurong kalahok kaugnay sa pagtuturo ng Mother Tongue PAGKATAPOS matanggap ang kopya ng nabuong Ortograpiyang Waray ng KWF.

Naging Madali. Ito ang temang nabuo sa ikalawang suliranin. Mapapansin sa mga tugon na madali na sa mga gurong kalahok ang pagtuturo ng Mother Tongue dahil sa ipinamahaging kopya ng KWF na naging daan para mas lalo pa nilang pagbutihin ang pagtuturo ng Mother Tongue na Waray. Natuklasan din sa mga tugon ng mga gurong kalahok na kung ang KWF ang mamamahagi ng mga materyales na magagamit nila sa pagtuturo ng Mother Tongue na Waray ay mas kampante sila dahil ito ang ahensiya na naatasan ng pamahalaan na pangalagaan ang mga katutubong wika, isa na rito, ang wikang Waray.

Tunghayan ang tugon ng mga gurong kalahok kaugnay sa karanasan nila sa pagtuturo ng Mother Tongue sa Baitang 3 pagkatapos makatanggap ng kopya ng Ortograpiyang waray mula sa KWF. Sa mga naging tugon ng mga gurong kalahok, mapapansin ang kanilang kasiyahan dahil sa pagkakaroon ng sariling kopya ng Ortograpiyang Waray na kanilang natanggap noong dumalo sila ng Panrehiyong Seminar at Paglulunsad ng Ortograpiyang Waray noong taong 2018. Dahil, nabuhayan sila ng loob lalo pa at ang materyal na kanilang naging batayan sa pagtuturo ng Mother Tongue na Waray partikular na sa tamang pagsulat at pagbigkas ng salita sa wikang Waray ay mula mismo sa ahensiya ng pamahalaan na KWF. Dahil dito, napatotohanan ang sinabi ni Jackson (2015) na kailangan magmula mismo sa kinauukulan ang pagpapatupad nito upang ganap na tanggapin ng mga mamamayan ang Ortograpiyang Waray. 
Mga karanasan ng guro sa baitang 3 sa pagtuturo ng mother tongue gamit ang Ortograpiyang Waray

Table 2

Pahayag ng kalahok sa temang naging madali pagkatapos matanggap ang Ortograpiyang Waray ng KWF

\begin{tabular}{lc}
\hline \multicolumn{1}{c}{ Tugon ng mga gurong Kalahok } & Tema \\
\hline Gumaan ang pakiramdam ko, sa wakas, kumilos na rin ang KWF na madali na ang pagtuturo. & Madali \\
Sa seminar pa lang natin, Nakita ko na sa ibingay niyong kopya mapapadali na ang pagtuturo. & Mapapadali \\
Natuwa at naiyak kasi may bibliya na kami upang madali nang ituro ang mother tongue. & Madali \\
Salamat sa KWF, nakatulong nang malaki ang Ortograpiyang Waray sa pagtuturo ng MT & Nakatulong \\
Mabuti nakadalo ako sa inyong Panrehiyong Seminar, nabigyan ako ng kopya, madali na. & Mapadali \\
Nakatulong nang labis para mapadali ang pagtuturo ng MT. & Nabawasan \\
Isang pagbati sa KWF, nabawasan din nang malaki ang paghihirap ko sa pagtuturo ng MT. & Madali \\
Utang na loob ko talaga sa KWF na nabigyan ako ng kopya, madali na ang pagtuturo ng MT.. & Nakatulong \\
Nakatulong nang malaki, kaya lang mga kaibigan ko, wala pang kopya & Madali \\
Kasama ko na ring itinuturo sa mga bata ang Ortograpiyang Waray. Madaling ituro ang MT. & Madali \\
Mayroon mga salita sa ortograpiya na iba ang kahulugan sa amin pero madali nang ituro. & Nakatulong \\
2018 pa 'yun sir, nakatulong nang malaki talaga, pero parang konti lang ang Leyte na salita. & Madali \\
Noong 2017, konsultasyon tapos 2018 namahagi na, ang galing ng KWF kaya madali na. & Madali \\
Madali nang ituro ang Mother Tongue, sana Diksiyunaryo naman sa Waray. & Madali \\
Madali nang magturo ng Mother Tongue (Waray) kasi may ortograpiya na. & \\
\hline
\end{tabular}

Ang huling suliranin ng pag-aaral na ito ay tungkol sa implikasyon ng pagkakaroon ng Ortograpiyang Waray na binuo ng KWF sa pagtuturo ng Mother Tongue. Bagama't ang implikasyon ay kasamang binanggit ng mga gurong kalahok, minabuti ng mananaliksik na banggitin muli ng mga gurong kalahok ang mga naging implikasyon ng pagkakaroon ng Ortograpiyang Waray sa pagtuturo ng Mother Tongue.

Table 3

Pahayag na tugon ng mga kalahok sa implikasyon sa pagkakaroon ng Ortograpiyang Waray ng KWF

\begin{tabular}{ll}
\multicolumn{1}{c}{ Tugon ng mga gurong Kalahok sa Implikasyon ng } & \multicolumn{1}{c}{ Implikasyon } \\
\hline Napagaan ang pagtuturo ng mother tongue. & Napaaan \\
Napadali na ang pagtuturo ng Mother Tongue. & Nabawasan \\
Nabawasan ang mga suliranin sa pagtuturo ng mother tongue. & Nakatulong \\
Nakatulong nang malaki sa pagtuturo ng mother tongue & Nakabuti \\
Nakabuti sa pagtuturo ng mother tongue & Nakagawa \\
Nakagawa ng sariling materyales sa pagtuturo ng mother tongue. & Nabuhayan \\
Nabuhayan ng loob sa pagtuturo ng mother tongue. & Napataas \\
Nagkaroon nang mataas na pagkilala sa pagtuturo ng mother tongue. & Lihitimo \\
Nagkaroon ng lihitimong batayan sa pagtuturo ng mother tongue. & Napalawak \\
Napalawak ang kaalaman sa pagtuturo ng mother tongue. & Nakapagbahagi \\
Nakapagbahagi sa kapwa guro ng kaalaman sa Ortograpiyang Waray. & Napalalim \\
Napalalim ang pag-unawa sa pagtuturo ng mother tongue. & Nakatulong \\
Nakatulong sa pagtuturo ng mother tongue. & Naisulong \\
Naisulong pang lalo ang pagtuturo ng mother tongue & Nagamit \\
Nagkaroon ng mga tinipong salita na magagamit sa pagtuturo ng mother tongue &
\end{tabular}

Batay sa mga naging tugon ng mga gurong kalahok kaugnay sa implikasyon ng pagkakaroon ng Ortograpiyang Waray sa pagtuturo ng Mother Tongue ay nakagawa ng sariling materyales (K6), nakapagbahagi sa kapwa guro ng kaalaman (K10), at nagkaroon ng mga tinipong salita (K14) na nagsagawa sila ng kanilang sariling pagsasanay o worksyap upang maituro nila nang maayos ang mother tongue na Waray (UNESCO 2019).

Kaugnay pa rito, sinabi rin ni Jackson (2015) na kung napaghandaan ng pamahalaan ay madali lamang ang pagtuturo ng mother tongue ayon sa mga sumusunod na tugon gaya ng nakabuti sa pagtuturo ng mother tongue (K5), nagkaroon ng lihitimong batayan (K8), at nagkaroon nang mataas na pagkilala sa pagtuturo ng mother tongue (K15) dahil sa proyento ng KWF na maglahtala ng Ortograpiyang Waray.

Gaya ng paniniwala ni Home (2015), ang ortograpiyang Filipino ay nagiging mabisa kung ang pagbigkas at bokabularyo nito ay nauunawaan ng bawat gumagamit ng wikang ito tulad ng tugon na napalawak ang kaalaman sa pagtuturo ng mother tongue (K9), napalalim ang pag-unawa sa pagtuturo ng mother tongue (K11), at nagsilbing bibliya sa pagtuturo ng mother tongue (K12). 
Samantala si Cabigao (2012), ay naniniwalang hindi magkakaroon ng problema ang isang ortograpiya partikular na sa Ortograpiyang Filipino kung ito ay ginagamit sa controlling domains gaya ng pamahalaan at iba pa. Napatunayan ito sa mga tugon na nakatulong nang malaki sa pagtuturo ng mother tongue (K4) at naisulong pang lalo ang pagtuturo ng mother tongue (K13).

Sa huli, Dagdag pa, bahagi ng mandato ng KWF ang pagbuo at pagsasaayos ng ortrograpiya ng mga katutubong wika sa Pilipinas na ginagamit ng mga tao (Corrige at KWF, 2018) kabilang na rito ang wikang Waray. Narito ang mga patunay na tugon tulad ng napagaan ang pagtuturo ng mother tongue (K1), napadali na ang pagtuturo ng Mother Tongue (K2), nabawasan ang mga suliranin sa pagtuturo ng mother tongue (K3) at nabuhayan ng loob sa pagtuturo ng mother tongue (K7).

\section{Konklusyon}

Batay sa resulta at pagtalakay sa isinagawang pag-aaral, nabuo ang mga sumusunod na konklusyon: Una, mahirap sa mga guro na nagtuturo ng Mother Tongue na Waray sa Baitang 3 nang wala pang legal na batayan ang tamang pagbigkas at pagsulat ng salita sa wikang katutubong Waray. Ikalawa, napadali at napagaan ang pagtuturo ng mga guro ng Mother Tongue na Waray sa Baitang 3 nang makatanggap ng sariling kopya mula sa ipinamahagi ng KWF. At, ikatlo, nakabuo ng mga kagamitang pampagtuturo ang mga guro na nagtuturo ng Mother Tongue na Waray mula nang magkaroon nang sariling kopya ng Ortograpiyang Waray.

Rekomendasyon - Mula sa konklusyon, nabuo ang mga sumusunod na rekomendasyon: Una, ipagpatuloy ang pagbuo ng mga kagamitang pampagtuturo na may kaugnayan sa pagtuturo ng Mother Tongue alinsunod sa mga nakapaloob sa Ortograpiyang Waray ng KWF. Ikalawa, magsagawa ng ibayong pagsasanay at worksyap sa bawat paaralan o distrito para sa ikauunlad pang lalo ng pagtuturo ng Mother Tongue na Waray at nang makapagprodyus nang marami pang kagamitang pampagtuturo. At, ikatlo, lahat ng modyul o ano pa mang kagamitang pampagtuturo ay kailangang nakaangkla sa Ortograpiyang Waray ng KWF dahil ito ang awtentikong batayan mula sa ahensiya ng pamahalaan.

\section{Sanggunian}

Cabigao, J. R. (2012). Saloobin at mungkahing pagbabago sa nilalaman ng 2009 gabay sa ortograpiyang filipino. Di-nalathalang Tesis. Pamantasang Normal ng Pilipinas, Maynila.

Corrige, M. V. G., \& Komisyon sa Wikang Filipino (KWF). (2018). Ortograpiya han waray. Komisyon sa wikang Filipino.

De Mesa, A. R. (2017). LNU-SWK terminal report 2017. LNU-IGP Graphics and Design.

De Mesa, A. R. (2018). LNU-SWK terminal report 2018. LNU-IGP Graphics and Design.

Del Ayre, J. O. Ortograpiyang pambansa. Retrieved June 18, 2021, from https://kwf.gov.ph/wp-content/uploads/Ortograpiyang_Pambansa_1.pdf

Home, C. M. (2015). Lawak ng kaalaman sa ortograpiyang filipino ng mga guro sa mataas na paaralan ng gubat, Distrito ng sorsogon. Tesis. Sorsogon State College.

Jackson, M.A (12 September 2015). Is your school ready for a mother tongue school program? Retrieved on April 12, 2021, from https://www.linkedin.com/pulse/your-school-ready-mother-tongue-programme-mattie-Jackson

UNESCO (04 June 2019). Improving the quality of mother tongue-based literacy and learning: Case studies from asia, africa and south america. Retrieved July 23, 2021, from https://bangkok.Unesco.org/content/improving-quality-mother-tongue-based-literacy-and-learning-casestudies-asia-africa-and

Zahavi, D. (2003) Husserl's phenomenology. Retrieved May 03, 2021, from https://books.google.com.ph/books/about/Husserl_s_Phenomenology.html?id=hZ3XAAAAMAAJ\&sou $\underline{\text { rce }=\mathrm{kp} \_ \text {book_description\&redir_esc }=y}$ 